\title{
On Statistical Convergence in Metric Spaces
}

\author{
Bilal Bilalov ${ }^{1}$, Tubu Nazarova ${ }^{1}$ \\ ${ }^{1}$ Institute of Mathematics and Mechanics of NAS of Azerbaijan, Baku, Azerbaijan. \\ Correspondence: Bilal Bilalov, Institute of Mathematics and Mechanics of NAS of Azerbaijan, 9, B.Vahabzade \\ Str., AZ 1141, Baku, Azerbaijan. E-mail: b_bilalov@mail.ru
}

Received: November 14, 2014 Accepted: December 17, 2014 Online Published: January 10, 2015

doi:10.5539/jmr.v7n1p37 URL: http://dx.doi.org/10.5539/jmr.v7n1p37

\begin{abstract}
The statistical convergence in metric spaces is considered. Its equivalence to the statistical fundamentality in complete metric spaces is proved. Introduced the concept of $p$-strong convergence, and proved its equivalence to the statistical convergence. Tauberian theorems concerning statistical convergence in metric spaces are given.
\end{abstract}

Keywords: statistical convergence, $p$-strong convergence, a metric space, Tauberian theorems

\section{Introduction}

The idea of statistical convergence was first proposed by A.Zigmund (Zygmund, 1979) in his famous monograph where he talked about "almost convergence". The first definition of it was given by H. Fast (Fast, 1951) and H. Steinhaus (Steinhaus, 1951). Later, this concept has been generalized in many directions. More details on this matter and on applications of this concept can be found in (T.C. Brown and A.R. Freedman, 1990; B.J. Connor, 1988; R. Erdös and G. Tenenbaum, 1989; A.R. Freedman and J.J. Sember, 1981; J.A. Fridy, 1985; J.A. Fridy and M.K. Khan, 1998; M. Kuchukaslan, U. Deger and O. Dovgoshey, 2012; M. Kuchukaslan and U. Deger, 2012; I.J. Maddox, 1988; D. Maharam, 1976; G.D. Maio and L.D.R. Kocinac, 2008; H.I.Miller, 1995; G.M. Peterson, 1966; I.J. Schoenberg, 1959). It should be noted that the methods of non-convergent sequences have long been known and they include e.g. Cesaro method, Abel method and etc. These methods are used in different areas of mathematics. For the applicability of these methods is very important that the considered space has a linear structure. Therefore, the study of statistical convergence in metric spaces is of special scientific interest. Different aspects of this problem is devoted in M. Kuchukaslan, U. Deger and O. Dovgoshey, 2014; M. Kuchukaslan and U. Deger, 2012. Statistical convergence is currently actively used in many areas of mathematics such as summation theory (B.J. Connor, 1988; A.R. Freedman and J.J. Sember, 1981; J.A. Fridy, 1985), number theory (R. Erdös and G. Tenenbaum, 1989), trigonometric series (A. Zygmund, 1979), probability theory (J.A. Fridy and M.K. Khan, 1998) measure theory (H.I. Miller, 1995), optimization (S. Pehlivan and M.A. Mamedov, 2000), approximation theory (A.D. Gadjiev and C. Orhan, 2002; A.D. Gadjiev, 2011), fuzzy theory, etc. Generalization of statistical convergence to the continuous case have done in (Bilalov, Sadigova).

It should be noted that the concept of statistical fundamentality (stat fundamentality) was first introduced by J.A. Fridy (J.A. Fridy, 1985) who proved its equivalence to statistical convergence with respect to numerical sequences. This problem was raised in (G.D. Maio and L.D.R. Kocinac, 2008) concerning uniform space $(X ; U)$. It is proved that if the sequence $\left\{x_{n}\right\}_{n \in \mathbb{N}} \subset X$ stat-convergent, then it is stat-fundamental. In the same paper raised the Problem 2.16 of the validity of converse statement.

In this paper we consider the statistical convergence in metric spaces. Statistical fundamentality is defined, and in a complete metric space it is proved that the statistical fundamentality is equivalent to the statistical convergence. Concept $p$-strong convergence in metric spaces is introduced and prove its equivalence to the one of statistical convergence. Some Tauberian theorems concerning statistical convergence in metric spaces are introduced. It should be noted that the issue of statistical convergence in metric spaces considered in (M. Kuchukaslan, U. Deger and O. Dovgoshey, 2014; M. Kuchukaslan and U. Deger, 2012). In these papers the statistical boundedness, the statistical equivalence of sequences in metric spaces and their relationship to the statistical convergence are considered. 


\section{Needful Information}

Let $(X ; \rho(\cdot ; \cdot))$ be a metric space with a metric $\rho$. Denote by $O_{\varepsilon}(a)$ the open ball in $X$ centered at the point $a \in X$ and with a radius $\varepsilon$ : $O_{\varepsilon}(a) \equiv\{x \in X: \rho(x ; a)<\varepsilon\}$. $A^{C}$ means the complement of the set $A \subset \mathbb{N}$ to $\mathbb{N}: A^{C}=\mathbb{N} \backslash A$, where $\mathbb{N}$ is a set of all positive numbers. $\chi_{A}(\cdot)$ is the characteristic function of $A ; \Rightarrow$ will be a quantifier which means "follows"; $\wedge$ will be a quantifier which means "and"; $\bar{M}$ will stand for the closure of $M$.

Let $A \subset \mathbb{N}$ be some set. Assume $\delta_{n}(A)=\frac{1}{n} \sum_{k=1}^{n} \chi_{A}(k)$. If $\exists \lim _{n \rightarrow \infty} \delta_{n}(A)=\delta(A)$, then $\delta(A)$ is called statistical density of the set $A$.

Accept the following

Definition 1. We say that $\left\{x_{n}\right\}_{n \in \mathbb{N}} \subset X$ statistically converges (st-converges) to $x \in X$, if $\delta\left(A_{\varepsilon}\right)=0$, where $A_{\varepsilon} \equiv\left\{n \in \mathbb{N}: \rho\left(x_{n} ; x\right) \geq \varepsilon\right\}$, and this kind of convergence is denoted as st- $\lim _{n \rightarrow \infty} x_{n}=x$.

Put

$$
\mathscr{K} \equiv\{K \subset \mathbb{N}: \delta(K)=1\} .
$$

In the sequel, we will need the following easily provable

Lemma 1. Let $K_{j} \in \mathscr{K}, j=1 ; 2 \Rightarrow K_{1} \cap K_{2} \in \mathscr{K}$.

Proof. In fact, let $I_{n} \equiv\{1 ; \ldots ; n\}$. We have

$$
K_{1} \cap K_{2}=\left(K_{1} \cup K_{2}\right) \backslash\left[\left(K_{2} \backslash K_{1}\right) \cup\left(K_{1} \backslash K_{2}\right)\right] .
$$

Consequently

$$
K_{1} \cap K_{2} \cap I_{n}=\left[\left(K_{1} \cup K_{2}\right) \cap I_{n}\right] \backslash\left[\left(\left(K_{2} \backslash K_{1}\right) \cup\left(K_{1} \backslash K_{2}\right)\right) \cap I_{n}\right] .
$$

As

$$
\left(\left(K_{2} \backslash K_{1}\right) \cup\left(K_{1} \backslash K_{2}\right)\right) \cap I_{n}=\left(\left(K_{2} \backslash K_{1}\right) \cap I_{n}\right) \cup\left(\left(K_{1} \backslash K_{2}\right) \cap I_{n}\right),
$$

taking into account

$$
\begin{gathered}
\left(K_{2} \backslash K_{1}\right) \cap I_{n} \subset K_{1}^{C} \cap I_{n} \Rightarrow \frac{\left|\left(K_{2} \backslash K_{1}\right) \cap I_{n}\right|}{\left|I_{n}\right|} \leq \frac{\left|K_{1}^{C} \cap I_{n}\right|}{\left|I_{n}\right|} \rightarrow 0, n \rightarrow \infty, \\
\frac{\left|\left(K_{1} \backslash K_{2}\right) \cap I_{n}\right|}{\left|I_{n}\right|} \rightarrow 0, n \rightarrow \infty,
\end{gathered}
$$

we get

$$
\frac{\left|\left(\left(K_{2} \backslash K_{1}\right) \cup\left(K_{1} \backslash K_{2}\right)\right) \cap I_{n}\right|}{\left|I_{n}\right|} \rightarrow 0, n \rightarrow \infty .
$$

From $\left(K_{1} \cap I_{n}\right) \subset\left(K_{1} \cup K_{2}\right) \cap I_{n}$ and $K_{1} \in \mathscr{K}$ it follows

$$
\frac{\left|\left(K_{1} \cup K_{2}\right) \cap I_{n}\right|}{\left|I_{n}\right|} \rightarrow 1, n \rightarrow \infty,
$$

and hence $K_{1} \cup K_{2} \in \mathscr{K}$. Then from (1) we directly obtain

$$
\frac{\left|K_{1} \cap K_{2} \cap I_{n}\right|}{\left|I_{n}\right|}=\frac{\left|\left(K_{1} \cup K_{2}\right) \cap I_{n}\right|}{\left|I_{n}\right|}-\frac{\left|\left(\left(K_{2} \backslash K_{1}\right) \cup\left(K_{1} \backslash K_{2}\right)\right) \cap I_{n}\right|}{\left|I_{n}\right|} \rightarrow 1, n \rightarrow \infty,
$$

i.e. $K_{1} \cap K_{2} \in \mathscr{K}$.

\section{Statistical Fundamentality}

Accept the following

Definition 2. We say that $\left\{x_{n}\right\}_{n \in \mathbb{N}} \subset X$ is statistically fundamental (st-fundamental) in $(X ; \rho)$, if $\forall \varepsilon>0$, $\exists n_{\varepsilon} \in \mathbb{N}: \delta\left(\Delta_{n_{\varepsilon}}\right)=0$, where

$$
\Delta_{n_{\varepsilon}} \equiv\left\{n \in \mathbb{N}: \rho\left(x_{n} ; x_{n_{\varepsilon}}\right) \geq \varepsilon\right\} .
$$

Let $x_{n} \stackrel{s t}{\rightarrow} x$ in $X$, and $\varepsilon>0$ be an arbitrary number. Put

$$
A_{\varepsilon} \equiv\left\{n: \rho\left(x_{n} ; x\right) \geq \varepsilon\right\} .
$$


It is absolutely clear that $\delta\left(A_{\varepsilon / 2}^{C}\right)=1$. Take $\forall n_{\varepsilon} \in A_{\varepsilon / 2}^{C}: \rho\left(x_{n_{\varepsilon}} ; x\right)<\frac{\varepsilon}{2}$. We have

$$
\left\{n: \rho\left(x_{n} ; x\right)<\frac{\varepsilon}{2}\right\} \subset\left\{n: \rho\left(x_{n} ; x_{n_{\varepsilon}}\right)<\varepsilon\right\},
$$

i.e. $A_{\varepsilon / 2}^{C} \subset \Delta_{n_{\varepsilon}}^{C}$, where $\Delta_{n_{\varepsilon}} \equiv\left\{n \in \mathbb{N}: \rho\left(x_{n} ; x_{n_{\varepsilon}}\right) \geq \varepsilon\right\}$. Hence, $\delta\left(\Delta_{n_{\varepsilon}}^{C}\right)=1 \Rightarrow \delta\left(\Delta_{n_{\varepsilon}}\right)=0$.

Thus, the following lemma is true.

Lemma 2. Let $x_{n} \stackrel{\text { st }}{\rightarrow} x$ in $(X ; \rho)$. Then the sequence $\left\{x_{n}\right\}_{n \in \mathbb{N}} \subset X$ is st-fundamental in $(X ; \rho)$.

Let $(X ; \rho)$ be complete metric space, and the sequence $\left\{x_{n}\right\}_{n \in \mathbb{N}} \subset X$ be st-fundamental in $(X ; \rho)$. Then $\exists n_{j} \in \mathbb{N}$ : $\delta\left(K_{j}\right)=1$, where $K_{j} \equiv\left\{n: \rho\left(x_{n} ; x_{n_{j}}\right) \leq 2^{1-j}\right\}, j=1,2$. By Lemma 1 we obtain $K_{1} \cap K_{2} \in \mathscr{K}$. Put

$$
M_{1} \equiv \overline{O_{1}\left(x_{n_{1}}\right)} \cap \overline{O_{2^{-1}}\left(x_{n_{2}}\right)} .
$$

It is obvious that $x_{n} \in M_{1}, \forall n \in\left(K_{1} \cap K_{2}\right) \equiv K_{(1)}$. Thus, $\exists n_{3} \in \mathbb{N}: K_{3} \in \mathscr{K}$, where $K_{3} \equiv\left\{n: \rho\left(x_{n} ; x_{n_{3}}\right) \leq 2^{-2}\right\}$. Let $K_{(2)}=K_{(1)} \cap K_{3}$. It is clear that $K_{(2)} \in \mathscr{K}$. Now let

$$
M_{2} \equiv M_{1} \cap \overline{O_{2^{-2}}\left(x_{n_{3}}\right)} .
$$

Denote by $d(M)$ the diameter of the set $M$, i.e.

$$
d(M)=\sup _{x, y \in M} \rho(x ; y) .
$$

Continuing in the same way, we obtain a sequence of closed sets $\left\{M_{n}\right\}_{n \in \mathbb{N}}, M_{1} \supset M_{2} \supset \ldots$, whose diameters tend to zero: $d\left(M_{n}\right) \leq 2^{-n+1} \rightarrow 0, n \rightarrow \infty$. Moreover, $K_{(n)} \in \mathscr{K}$, where $K_{(n)} \equiv\left\{k \in \mathbb{N}: x_{k} \in M_{n}\right\}$. Take $\forall \tilde{x}_{n} \in M_{n}$. We have

$$
\rho\left(\tilde{x}_{n} ; \tilde{x}_{n+p}\right) \leq d\left(M_{n}\right) \rightarrow 0, n \rightarrow \infty, \forall p \in \mathbb{N} .
$$

Hence, the sequence $\left\{\tilde{x}_{n}\right\}_{n \in \mathbb{N}}$ is fundamental in $(X ; \rho)$ and as $(X ; \rho)$ is complete, it is clear that $\exists x \in X: \tilde{x}_{n} \rightarrow$ $x, n \rightarrow \infty$. It is absolutely clear that $x \in \bigcap_{n} M_{n}$, i.e. $\cap_{n} M_{n}$ is non-empty. From $d\left(M_{n}\right) \rightarrow 0, n \rightarrow \infty$, it directly follows that $\{x\}=\bigcap_{n} M_{n}$, i.e. $\cap_{n} M_{n}$ consists of one element. As $K_{(m)} \in \mathscr{K}$, then $\exists\left\{n_{m}\right\}_{m \in \mathbb{N}} \subset \mathbb{N} ; n_{1}<n_{2}<\ldots$ :

$$
\frac{1}{n}\left|\left\{k \in I_{n}: k \in K_{(m)}^{C}\right\}\right|<\frac{1}{m}, \forall n>n_{m},
$$

where $I_{n} \equiv\{1 ; \ldots ; n\}$. Assume

$$
\mathbb{N}_{0} \equiv\left\{k \in \mathbb{N}: n_{m}<k \leq n_{m+1} \wedge k \in K_{(m)}^{C}\right\}
$$

and

$$
y_{k}=\left\{\begin{array}{l}
x, \text { if } k \in \mathbb{N}_{0} \wedge\left(k>n_{1}\right) \\
x_{k}, \text { if othervise. }
\end{array}\right.
$$

Take $\forall \varepsilon>0$. If $k \in \mathbb{N}_{0} \wedge\left(k>n_{1}\right)$, then $y_{k}=x$, and, as a result $0=\rho\left(y_{k} ; x\right)<\varepsilon$. If $k \notin \mathbb{N}_{0} \Rightarrow k \in K_{(m)} \Rightarrow x_{k} \in$ $M_{m} \Rightarrow \rho\left(x_{k} ; x\right) \leq \rho\left(x_{k} ; x_{n_{m}}\right)+\rho\left(x_{n_{m}} ; x\right) \leq 2^{-m+2}<\varepsilon$, for sufficiently great values of $m$. Consequently, $\lim _{k \rightarrow \infty} y_{k}=x$.

Let $\tilde{K} \equiv\left\{k \in \mathbb{N}: x_{k} \neq y_{k}\right\}$. Let us show that $\delta(\tilde{K})=0$. Put $n_{m}<n<n_{m+1}$. Let us prove that

$$
\left\{k \leq n: x_{k} \neq y_{k}\right\} \subset\left\{k \leq n: k \in K_{(m)}^{C}\right\} .
$$

Let $k \leq n \wedge x_{k} \neq y_{k}$. Consequently, $k \in \mathbb{N}_{0} \Rightarrow k \in K_{(m)}^{C}$. Thus

$$
\frac{1}{n}\left|\left\{k \leq n: x_{k} \neq y_{k}\right\}\right| \leq \frac{1}{n}\left|\left\{k \leq n: k \in K_{(m)}^{C}\right\}\right|<\frac{1}{m} .
$$

It is clear that if $n \rightarrow \infty$, then $m \rightarrow \infty$. Then from the previous relation we get

$$
\lim _{n \rightarrow \infty} \frac{\left|\left\{k \leq n: x_{k} \neq y_{k}\right\}\right|}{n}=0 .
$$


Consequently, $\left\{k \leq n: x_{k} \neq y_{k}\right\}^{C} \in \mathscr{K}$ and $\lim _{n \rightarrow \infty} y_{n}=x$. Let us show that $s t$ - $\lim _{n \rightarrow \infty} x_{n}=x$. Take $\forall \varepsilon>0$. We have

$$
\left\{k \leq n: \rho\left(x_{k} ; x\right) \geq \varepsilon\right\} \subset\left\{k \leq n: x_{k} \neq y_{k}\right\} \cup\left\{k \leq n: \rho\left(y_{k} ; x\right) \geq \varepsilon\right\} .
$$

As, $\lim _{k \rightarrow \infty} y_{k}=x$ in $(X ; \rho)$, then $\exists n_{\varepsilon} \in \mathbb{N}: \rho\left(y_{k} ; x\right)<\varepsilon, \forall k \geq n_{\varepsilon}$. Consequently

$$
\left|\left\{k \leq n: \rho\left(y_{k} ; x\right) \geq \varepsilon\right\}\right| \leq n_{\varepsilon} \Rightarrow \frac{1}{n}\left|\left\{k \leq n: \rho\left(y_{k} ; x\right) \geq \varepsilon\right\}\right| \leq \frac{n_{\varepsilon}}{n} \rightarrow 0, n \rightarrow \infty .
$$

Then, using (2), from (3) we obtain

$$
\begin{gathered}
\frac{1}{n}\left|\left\{k \leq n: \rho\left(x_{k} ; x\right) \geq \varepsilon\right\}\right| \leq \frac{1}{n}\left|\left\{k \leq n: x_{k} \neq y_{k}\right\}\right|+ \\
+\frac{1}{n}\left|\left\{k \leq n: \rho\left(y_{k} ; x\right) \geq \varepsilon\right\}\right| \rightarrow 0, n \rightarrow \infty .
\end{gathered}
$$

So, $s t-\lim _{n \rightarrow \infty} x_{n}=x$. Thus, we have proved the following theorem.

Theorem 1. Let $(X ; \rho)$ be a complete metric space and $\left\{x_{n}\right\}_{n \in \mathbb{N}} \subset X$ be some system. Then the following statements are equivalent to each other:

1) $\exists s t-\lim _{n \rightarrow \infty} x_{n}$;

2) $\left\{x_{n}\right\}_{n \in \mathbb{N}}$ is st-fundamental;

3) $\exists\left\{y_{n}\right\}_{n \in \mathbb{N}} \subset X: \exists \lim _{n \rightarrow \infty} y_{n} \wedge\left\{n \in \mathbb{N}: x_{n}=y_{n}\right\} \in \mathscr{K}$.

This theorem immediately implies the following

Corollary 1. Let $\left\{x_{n}\right\}_{n \in \mathbb{N}} \subset X$ and $\exists s t-\lim _{n \rightarrow \infty} x_{n}=x$. Then $\exists\left\{n_{k}\right\}_{k \in \mathbb{N}} \subset \mathbb{N}: n_{1}<n_{2}<\ldots, \lim _{k \rightarrow \infty} x_{n_{k}}=x \wedge \delta\left(\left\{n_{k}\right\}_{k \in \mathbb{N}}\right)=$ 1.

\section{4. $p$-strong Convergence}

Let $(X ; \rho)$ be a metric space, and $p \in(0,+\infty)$ be some number. Following (A. Alotaibi and A.M. Alroqi, 2012) we accept the following

Definition 3. The sequence $\left\{x_{n}\right\}_{n \in \mathbb{N}} \subset X$ is called p-strong convergent to $x \in X$, if $\lim _{n \rightarrow \infty} \frac{1}{n} \sum_{k=1}^{n} \rho^{p}\left(x_{k} ; x\right)=0$, and this kind of limit is denoted as $p$ - $\lim _{n \rightarrow \infty} x_{n}=x$.

The following theorem is true.

Theorem 2. It holds: i) If $p-\lim _{n \rightarrow \infty} x_{n}=x$ then $\exists s t-\lim _{n \rightarrow \infty} x_{n} \wedge s t-\lim _{n \rightarrow \infty} x_{n}=x$; ii) If $\exists s t-\lim _{n \rightarrow \infty} x_{n}=x$ and $\exists O_{r}\left(x_{0}\right) \subset X$ : $x_{n} \in O_{r}\left(x_{0}\right), \forall n \in \mathbb{N}$, then $\exists p-\lim _{n \rightarrow \infty} x_{n}=x$.

Proof. i) Let $p$ - $\lim _{n \rightarrow \infty} x_{n}=x$. Take $\forall \varepsilon>0$, and put $K_{\varepsilon}^{(n)}=\left\{n \in I_{n}: \rho\left(x_{n} ; x\right) \geq \varepsilon\right\}$. We have

$$
\varepsilon^{p} \frac{\left|K_{\varepsilon}\right|}{n} \leq \frac{1}{n} \sum_{k \in K_{\varepsilon}^{(n)}} \rho^{p}\left(x_{k} ; x\right) \leq \frac{1}{n} \sum_{k=1}^{n} \rho^{p}\left(x_{k} ; x\right) \rightarrow 0, n \rightarrow \infty,
$$

i.e. $\delta\left(K_{\varepsilon}\right)=0 \Rightarrow s t$ - $\lim _{n \rightarrow \infty} x_{n}=x$.

ii) Let $\exists s t-\lim _{n \rightarrow \infty} x_{n}=x$ and $\exists O_{r}\left(x_{0}\right) \subset X: x_{n} \in O_{r}\left(x_{0}\right), \forall n \in \mathbb{N}$. We have

$$
\rho\left(x_{n} ; x\right) \leq \rho\left(x_{n} ; x_{0}\right)+\rho\left(x_{0} ; x\right) \leq r+\rho\left(x_{0} ; x\right)=M .
$$

Thus

$$
\frac{1}{n} \sum_{k=1}^{n} \rho^{p}\left(x_{k} ; x\right)=\frac{1}{n} \sum_{k \in I_{n} \backslash K_{\varepsilon}^{(n)}} \rho^{p}\left(x_{k} ; x\right)+\frac{1}{n} \sum_{k \in K_{\varepsilon}} \rho^{p}\left(x_{k} ; x\right),
$$


where $K_{\varepsilon}^{(n)}=\left\{k \in I_{n}: \rho\left(x_{k} ; x\right) \geq \varepsilon\right\}, \varepsilon>0$ is an arbitrary number. So

$$
\begin{gathered}
\frac{1}{n} \sum_{k \in I_{n} \backslash K_{\varepsilon}^{(n)}} \rho^{p}\left(x_{k} ; x\right) \leq \varepsilon^{p} ; \\
\frac{1}{n} \sum_{k \in K_{\varepsilon}} \rho^{p}\left(x_{k} ; x\right) \leq M^{p} \frac{\left|K_{\varepsilon}\right|}{n} \rightarrow 0, n \rightarrow \infty .
\end{gathered}
$$

From the arbitrariness of $\varepsilon$ we obtain that

$$
\frac{1}{n} \sum_{k=1}^{n} \rho^{p}\left(x_{k} ; x\right)=0 \Rightarrow p-\lim _{n \rightarrow \infty} x_{n}=x .
$$

Definition 4. The function $\mu:[0, \infty) \rightarrow[0, \infty)$ is called a modulus, if: (i) $\mu(0)=0 \Leftrightarrow x=0$; (ii) $\mu(x+y) \leq$ $\mu(x)+\mu(y), \forall x, y \in[0, \infty) ;$ (iii) $\mu$ is a monotone nondecreasing function; (iv) $\mu(+0)=0$.

According to (I.J. Maddox, 1988) we accept the following

Definition 5. Let $(X ; \rho)$ be a metric space and $\mu$ be a modulus. $\left\{x_{n}\right\}_{n \in \mathbb{N}} \subset X$ is called $\mu$-convergent to $x \in X$, if

$$
\lim _{n \rightarrow \infty} \frac{1}{n} \sum_{k=1}^{n} \mu\left(\rho\left(x_{k} ; x\right)\right)=0,
$$

and this kind of convergence is denoted as $\mu$ - $\lim _{n \rightarrow \infty} x_{n}=x$.

Similarly to Theorem 2 we prove the following

Theorem 3. Let $\exists \mu-\lim _{n \rightarrow \infty} x_{n}=x$. Then $\exists s t-\lim _{n \rightarrow \infty} x_{n} \wedge s t$ - $\lim _{n \rightarrow \infty} x_{n}=x$.

\section{Tauberian Theorems in Metric Spaces}

Let $(X ; \rho)$ be a metric space and $\left\{x_{n}\right\}_{n \in \mathbb{N}} \subset X$ be some sequence. Let $\Delta \rho_{n}=\rho\left(x_{n} ; x_{n+1}\right), \forall n \in \mathbb{N}$. The following theorem is true.

Theorem 4. Let st $\lim _{n \rightarrow \infty} x_{n}=x$ and $\Delta \rho_{n}=\overline{\bar{o}}\left(\frac{1}{n}\right)$. Then $\exists \lim _{n \rightarrow \infty} x_{n} \wedge \lim _{n \rightarrow \infty} x_{n}=x$.

Proof. We will follow (J.A. Fridy, 1985). Let $s t-\lim _{n \rightarrow \infty} x_{n}=x$. Then, by Theorem $1 \exists\left\{y_{n}\right\}_{n \in \mathbb{N}} \subset X: \lim _{n \rightarrow \infty} y_{n}=$ $x \wedge\left\{n: x_{n}=y_{n}\right\} \in \mathscr{K}$. Every $k \in \mathbb{N}$ can be represented as $k=m_{k}+p_{k}$, where

$$
m_{k}=\left\{\begin{array}{l}
\max \left\{i \leq k: x_{i}=y_{i}\right\}, \quad A_{k} \neq \emptyset, \\
-1, \quad A_{k}=\emptyset
\end{array}\right.
$$

$A_{k}=\left\{i \leq k: x_{i}=y_{i}\right\}$. As proved in (A.R. Freedman and J.J. Sember, 1981), it holds that $\lim _{k \rightarrow \infty} \frac{p_{k}}{m_{k}}=0$. It is clear that $\exists M>0: \Delta \rho_{n} \leq \frac{B}{n}, \forall n \in \mathbb{N}$.

We have

$$
\begin{gathered}
\rho\left(y_{m_{k}} ; x_{k}\right)=\rho\left(x_{m_{k}} ; x_{k}\right)=\rho\left(x_{m_{k}} ; x_{m_{k}+p_{k}}\right) \leq \\
\leq \sum_{i=m_{k}}^{m_{k}+p_{k}-1} \rho\left(x_{i} ; x_{i+1}\right) \leq \sum_{i=m_{k}}^{m_{k}+p_{k}-1} \Delta \rho_{i} \leq M \frac{p_{k}}{m_{k}} \rightarrow 0, k \rightarrow \infty .
\end{gathered}
$$

As, $\lim _{k \rightarrow \infty} y_{m_{k}}=x$ in $(X ; \rho)$, it directly follows that $\lim _{k \rightarrow \infty} x_{k}=x$.

We say that $\left\{x_{k}\right\}_{k \in \mathbb{N}} \subset X$ is a gap sequence if $\Delta \rho_{k}=0$ except for certain indices $k$ which occur at wide intervals or gaps.

The following Tauberian theorem is true.

Theorem 5. Let $\{k(i)\}_{i \in \mathbb{N}} \subset \mathbb{N}$ be an increasing sequence such that $\lim \inf _{i} \frac{k(i+1)}{k(i)}>1$ and let $\left\{x_{k}\right\}_{k \in \mathbb{N}} \subset X$ be corresponding gap sequence: $\Delta \rho_{n}=0$ if $k \neq k(i)$ for $i \in \mathbb{N}$. If st $\lim _{n \rightarrow \infty} x_{n}=x$, then $\lim _{n \rightarrow \infty} x_{n}=x$. 
Proof. Quite similarly proved in (J.A. Fridy, 1985) For completeness of the exposition we present it here. Let $\liminf \sin _{i} \frac{k(i+1)}{k(i)}=1+2 \delta>1$. Then for sufficiently great values of $i$ we have

$$
\frac{k(i+1)}{k(i)}>1+\delta>1,
$$

i.e.

$$
k(i+1)-k(i)>\delta k(i) .
$$

This means that the number of terms in the $(i+1)$-st block (throughout which $x_{k}$ is constant) is greater than $\delta k(i)$. Now, let us assume that $\lim _{n \rightarrow \infty} x_{n} \neq x$. Take $\varepsilon>0$. Let $k \in \mathbb{N}$ be sufficiently great, such that $\rho\left(x_{k} ; x\right) \geq \varepsilon$. Thus if such a $k$ is chosen from the $(i+1)$-st block, where $i$ is large enough to ensure that (4) holds, we have

$$
\frac{1}{k(i+1)}\left|\left\{k \leq k(i+1): \rho\left(x_{k} ; x\right) \geq \varepsilon\right\}\right|>\frac{k(i+1)-k(i)}{k(i+1)}>\frac{\delta}{1+\delta} .
$$

Thus, $\frac{1}{n}\left|\left\{k \leq n: \rho\left(x_{k} ; x\right) \geq \varepsilon\right\}\right|$ does not tend to zero, so $s t$ - $\lim _{n \rightarrow \infty} x_{n} \neq x$.

\section{References}

Alotaibi, A., \& Alroqi, A. M. (2012). Statistical convergence in a paranormed space. Journal of Inequalities and Applications, 39, 1-6.

Bilalov, B. T., \& Sadigova, S. R. On $\mu$-statistical convergence. Proceedings of the American Mathematical Society; accepted paper.

Brown, T. C., \& Freedman, A. R. (1990). The uniform density of sets of integers and Fermat's last Theorem. Compt. Rendus Math. L'Acad. Sci, 12, 1-6.

Connor, B. J. (1988). The statistical and strong p-Cesaro convergence of sequences. Analysis, 8, 47-63. http://dx.doi.org/10.1524/anly.1988.8.12.47

Erdös, R., \& Tenenbaum, G. (1989). Sur les densities de certaines suites dentiers. Proc. London Math. Soc., 59(3), 417-438. http://dx.doi.org/10.1112/plms/s3-59.3.417

Fast, H. (1951). Sur la convergence statistique. Colloquium Mathematicum, 2, 241-244.

Freedman, A. R., \& Sember, J. J. (1981). Densities and summability. Pacific J. of Math., 95(2), $293-305$. http://dx.doi.org/10.2140/pjm.1981.95.293

Fridy, J. A. (1985). On statistical convergence. Analysis, 5, 301-313. http://dx.doi.org/10.1524/anly.1985.5.4.301

Fridy, J. A., \& Khan, M. K. (1998). Tauberian theorems via statistical convergence J. of Math. Anal. and Appl., 228(1), 73-95. http://dx.doi.org/10.1006/jmaa.1998.6118

Gadjiev, A. D., \& Orhan, C. (2002). Some approximation theorems via statistical convergence. Rocky Mountain Jour. of Math., 32(1), 129-138. http://dx.doi.org/10.1216/rmjm/1030539612

Gadjiev, A. D. (2011). Simultaneous statistical approximation of analytic functions and their derivatives by $k$ positive linear operators. Azerb. J. of Math., 1(1), 57-66.

Kuchukaslan, M., Deger, U., \& Dovgoshey, O. (2014). On the statistical convergence of metric valued sequences. Ukrainian Mathematical Journal, 66(5), 796-805. http://dx.doi.org/10.1007/s11253-014-0974-z

Kuchukaslan, M., \& Deger, U. (2012). On statistical boundedness of metric valued sequences. European Journal of Pure and Applied Mathematics, 5(2), 174-186.

Maddox, I. J., (1988). Statistical convergence in a locally convex space. Math. Proc. of the Cambridge, Phil. Soc., 104, 141-145. http://dx.doi.org/10.1017/S0305004100065312

Maharam, D. (1976). Finitely additive measures on the integers. Sankhya Ser, 38, 44-59.

Maio, G. D., \& Kocinac, L. D. R. (2008). Statistical convergence in topology. Topology and its Applications, 156, 28-45. http://dx.doi.org/10.1016/j.topol.2008.01.015

Miller, H. I. (1995) A measure theoretical subsequence characterization of statistical convergence. Transactions of the American Mathematical Society, 347(5), 1811-1819. 
Pehlivan, S., \& Mamedov, M. A. (2000). Statistical cluster points and turnpike. Optimization, 48(1), 91-106.

Peterson, G. M. (1966). Regular Matrix Tramformations. Mc Graw-Hill, London-New York-Toronto-Sydney.

Schoenberg, I. J. (1959). The integrability of certain functions and related summability methods. Amer. Math. Monthly, 66, 361-375.

Steinhaus, H. (1951). Sur la convergence ordinaire et la convergence asymptotique. Colloquium Mathematicum, 2, 73-74.

Zygmund, A. (1979). Trigonometric series. Cambridge UK, $2^{\text {nd }}$ edition.

\section{Copyrights}

Copyright for this article is retained by the author(s), with first publication rights granted to the journal.

This is an open-access article distributed under the terms and conditions of the Creative Commons Attribution license (http://creativecommons.org/licenses/by/3.0/). 